\title{
Condition Monitoring Method for Automatic Transmission Clutches
}

\author{
Agusmian Partogi Ompusunggu ${ }^{1}$, Jean-Michel Papy ${ }^{2}$, Steve Vandenplas $^{3}$, Paul Sas $^{4}$, and Hendrik Van Brussel ${ }^{5}$ \\ 1,2,3 Flanders' Mechatronics Technology Centre (FMTC), Celestijnenlaan 300D, 3001 Heverlee, Belgium. \\ agusmian.ompusunggu@fmtc.be \\ jean-michel.papy@fmtc.be \\ steve.vandenplas@fmtc.be \\ ${ }^{4,5}$ K.U.Leuven, Department of Mechanical Engineering, Division PMA, Celestijnenlaan 300B, 3001 Heverlee, Belgium. \\ paul.sas@mech.kuleuven.be \\ hendrik.vanbrussel@mech.kuleuven.be
}

\begin{abstract}
This paper presents the development of a condition monitoring method for wet friction clutches which might be useful for automatic transmission applications. The method is developed based on quantifying the change of the relative rotational velocity signal measured between the input and output shaft of a clutch. Prior to quantifying the change, the raw velocity signal is preprocessed to capture the relative velocity signal of interest. Three dimensionless parameters, namely the normalized engagement duration, the normalized Euclidean distance and the spectral angle mapper distance, that can be easily extracted from the signal of interest are proposed in this paper to quantify the change. In order to experimentally evaluate and verify the potential of the proposed method, clutches' life data obtained by conducting accelerated life tests on some commercial clutches with different lining friction materials using a fully instrumented SAE\#2 test setup, are utilized for this purpose. The aforementioned parameters extracted from the experimental data clearly exhibit progressive changes during the clutch service life and are well correlated with the evolution of the mean coefficient of friction (COF), which can be seen as a reference feature. Hence, the quantities proposed in this paper can therefore be seen as principle features that may enable us to monitor and assess the condition of wet friction clutches.
\end{abstract}

\section{INTRODUCTION}

Vehicles equipped with automatic transmissions have gained popularity in recent years. As is obvious from its name, an automatic transmission is a transmission which shifts power or speed by itself. In this kind of transmissions, wet friction

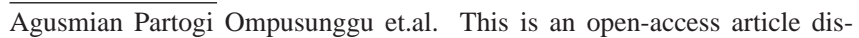
tributed under the terms of the Creative Commons Attribution 3.0 United States License, which permits unrestricted use, distribution, and reproduction in any medium, provided the original author and source are credited.
}

clutches are one of critical components that play a major role on the performance.

Wet friction clutches are machine elements enabling the power transmission from an input shaft (driving side) to an output shaft (driven side) during the operation, based on the friction occurring on lubricated contacting surfaces. The contacting surfaces comprise friction surface (friction disc) and counter surface (separator disc). The clutch is lubricated by an automatic transmission fluid (ATF) having a main function as a cooling lubricant cleaning the contacting surfaces and giving smoother performance and longer life. Besides for the clutch lubrication, this oil is also used for the clutch actuation.

The presence of the ATF in the clutch, however, reduces the coefficient of friction (COF). In applications where high power is mandatory, e.g. heavy duty vehicles (tractors, harvesters, etc), the clutch is therefore designed with multiple friction and separator discs. This configuration is known as a multi-disc wet friction clutch as schematically shown in Figure 1 , in which the friction discs are mounted to the hub by splines, and the separator discs are mounted to the drum by lugs. The friction disc is made of a steel-core disc with lining friction material bonded on both sides and the separator disc is made of plain steel. In addition, the input shaft is commonly connected to the drum side, while the output shaft is connected to the hub side.

An electro-mechanical-hydraulic actuator is commonly used for both disengagement and engagement mechanisms of wet friction clutches. This actuator consists of some main components, such as: a piston, a returning spring which is always under compression and a hydraulic group consisting of a control valve, an oil pump, a filter, etc. Figure 1 shows the assembly of the piston and the returning spring in the interior of a wet friction clutch. To engage a wet friction clutch, a pressur- 


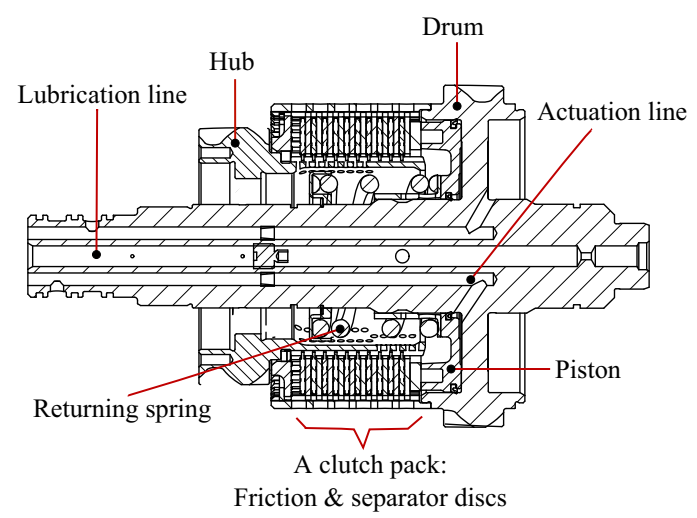

(a)

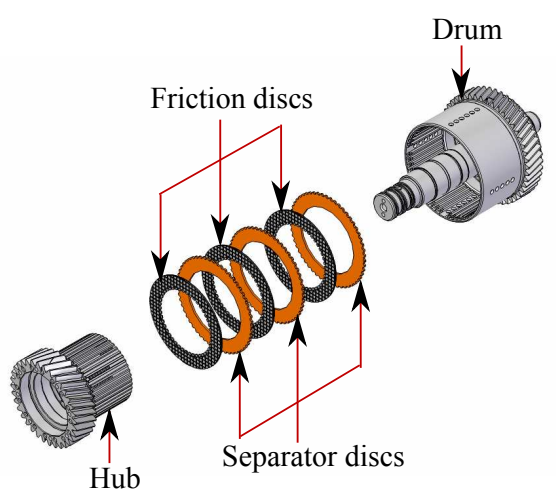

(b)

Figure 1. Configuration of a multi-disc wet friction clutch, (a) cross-sectional and (b) exploded view.

ized ATF actuated by the control valve is applied through the actuation line in order to generate a force acting on the piston. When the applied pressure exceeds a certain value to overcome the resisting force arising from both spring force and frictional force occurring between the piston and the internal surface of the drum, the piston starts moving and eventually pushes both friction and separator discs toward each other. To disengage the clutch, the pressurized ATF is released such that the returning spring is allowed to push the piston back to its rest position.

An unexpected failure occurring in the clutch can therefore lead to total breakdown of the vehicles. The impact can put human safety at risk, possibly cause long term vehicle down times, and result in high maintenance costs. In order to minimize the negative impacts caused by an unexpected breakdown, an optimal maintenance strategy driven by an accurate condition monitoring and prognostics needs to be applied for wet friction clutches. Although they are critical components, to our knowledge very little attention has been paid to these particular components in terms of the development of a condition monitoring method (tool). The main objective of this study is to develop a condition monitoring method for wet friction clutches that can be practically used in real-life applications. Furthermore, the developed method can be possibly extended towards clutch condition prognosis, but this is out of the scope of the paper.

Feature extraction is a key step to succeed in the development of a condition monitoring method. A feature can be derived based on the physics of degradation of the case of interest or based on heuristic (data-driven) approach. The derivation of physics-based features requires a profound understanding about the physics of degradation, while the derivation of heuristic-based features requires a large number of training data and experience about the case of interest. Furthermore, a parameter or quantity can be considered as a principle feature if it effectively delivers useful information about the failure mode and level. In general, the evolution of (a combination of) principle features can be associated with the progress of a target failure.

The coefficient of friction (COF), which can be seen as a physics-based feature, has been used for many years as a principle feature for monitoring the condition of wet friction clutches (Matsuo \& Saeki, 1997; Ost, Baets, \& Degrieck, 2001; Maeda \& Murakami, 2003; Li et al., 2003; Fei, Li, Qi, $\mathrm{Fu}, \& \mathrm{Li}, 2008)$. However, the use of the COF for clutch monitoring is possibly expensive and not easily implementable for real-life applications, due to the fact that at least two sensors are required to extract the COF, namely (i) a torque sensor and (ii) a force sensor, which are commonly difficult to install in a transmission (i.e. typically not available in today's transmissions). The quasi-steady-state clutch torque may be estimated from the torque-velocity characteristics of engine/torque converter, while the normal (axial) clutch force may be approximated from the pressure applied on the piston. However, the torque and normal force estimations with this approach can lead to inaccurate COF estimation.

Furthermore, several methods have been proposed in literature for assessing the condition of wet friction clutches based on the quality of the friction material, namely (i) Scanning Electron Microscope (SEM) micrograph, (ii) surface topography, (iii) Pressure Differential Scanning Calorimetry (PDSC) and (iv) Attenuated Total Reflectance Infrared spectroscopy (ATR-IR) (Jullien, Meurisse, \& Berthier, 1996; Guan, Willermet, Carter, \& Melotik., 1998; Li et al., 2003; Maeda \& Murakami, 2003; Nyman, Maki, Olsson, \& Ganemi, 2006). Nevertheless, these methods are not practically implementable during operation, owing to the fact that the friction discs have to be taken out from the clutch pack and then prepared for assessing the degradation level. In other words, an online condition monitoring system can not be realized by using these existing methods.

In our recent studies (Ompusunggu, Papy, Vandenplas, Sas, \& VanBrussel, 2009; Ompusunggu, Sas, VanBrussel, AlBender, Papy, \& Vandenplas, 2010; Ompusunggu, Sas, Van- 
Brussel, Al-Bender, \& Vandenplas, 2010; Ompusunggu, Sas, et al., 2011; Ompusunggu, Papy, Vandenplas, Sas, \& VanBrussel, n.d.), some potential features extracted from the preand post-lockup torsional vibration and normal-mode vibration signals, have been investigated and proven to be relevant for condition monitoring of wet friction clutches. These potential features are derived based on both physical and heuristic reasonings. Although they have potential as features for clutch monitoring, determining principle features that are robust, relatively easy to measure and inexpensive to compute for clutch condition monitoring remains a subject for further investigation.

As the degradation occurring in wet friction clutches progresses, the frictional characteristics change which consequently alters the behavior and performance of clutches. Based on this reasoning, it is hypothesized that the change of clutch behavior is also reflected by the change of the relative rotational velocity signal measured between the input and output shaft of the clutch. This hypothesis has been confirmed theoretically and experimentally in another recent study (Ompusunggu, Janssens, Al-Bender, Sas, \& VanBrussel, 2011). In this present paper, the development of a condition monitoring method for wet friction clutches based on monitoring the change of the relative velocity signal is presented. Three parameters, namely the engagement duration and two dissimilarity measures, namely the Euclidean distance and Spectral Angle Mapper (SAM) distance (Kruse et al., 1993; Paclik \& Duin, 2003) are proposed in this paper as features for clutch monitoring. In order to experimentally evaluate and verify the potential of the proposed method, clutches' life data obtained by conducting accelerated life tests on some commercial clutches with different lining friction materials using a fully instrumented SAE\#2 test setup, are utilized for this purpose. In the tests, the COF is measured and used as a reference feature to evaluate the relevance of the proposed features.

The remainder of this paper is organized as follows. After introducing the objective and motivation, the methodology of clutch monitoring developed in this study is presented and discussed in Section 2. Service life data of some clutches obtained from accelerated life tests carried out on the used SAE\#2 test setup are employed for the evaluation of the method, where the experimental aspects are described in Section 3. The results obtained after applying the proposed method to the clutches' life data are further presented and discussed in Section 4. Finally, some conclusions drawn from the study are presented in Section 5.

\section{Methodology}

Background that motivates the development of the methodology is first discussed in this section. Then, the signal preprocessing technique prior to computing the proposed features is described. Finally, the formulas to compute the features are presented and discussed.

\subsection{Background}

As reported in literature, degradation occurring in wet friction clutches (e.g. friction materials) alters the frictional characteristics. A change of the frictional characteristics during the clutches' service life is exposed by a decreasing coefficient of friction (COF) (Ost et al., 2001; Fei et al., 2008). With the same operational condition, this decreasing COF implies that the torque transmitted during clutch engagement drops. Besides a COF reduction, another aspect which is often associated with the change of the clutch frictional characteristics is the loss of anti-shudder property. Due to this, the damping characteristic of the clutch system changes (the equivalent damping can be negative). It is commonly accepted that the loss of anti-shudder property in a clutch can lead to the occurrence of the stick-slip or/and self excited vibration in the driveline.

As a result of the change of the frictional characteristics, the dynamic responses of clutches during and after the engagement phase also change. In this study, the relative velocity profile measured between the input and output shafts of a clutch, which can be seen as a representation of the dynamic behavior during the engagement phase, is considered as the one that is significantly affected by the change of the clutch frictional characteristics. It is important to note that the use of the latter signal is motivated by the fact that it is possible to measure (online) in real-life applications since the rotational velocity sensors (e.g. Hall-effect encoders) are typically available in automatic transmissions.

\subsection{Capturing the signal of interest}

Prior to computing the features, the raw signals obtained from measurements first need to be preprocessed. Figure 2 graphically illustrates the signal preprocessing step, namely the procedure to capture the relative rotational velocity signal of interest based on two raw signals: (i) the relative rotational velocity signal and (ii) pressure applied to the clutch piston signal. Once the signal of interest is captured using the two raw signals, features can then be extracted for clutch monitoring purpose.

Let the signal of interest be captured at a given duty cycle with a predetermined time record length $\tau$, and suppose that the time record length is kept the same for all duty cycles. For the sake of consistency, the signal is always captured at the same reference time instant. It is reasonable to consider the time instant when the ATF pressure applied to the clutch pack $p(t)$ starts to increase from zero value $t_{f}$ as the reference time instant. For an ideal pressure signal, i.e. continuous and noiseless, $t_{f}$ can be mathematically formulated as:

$$
t_{f}=\min \{\forall t \in \mathbb{R}: \quad p(t)>0\} .
$$


While the applied pressure is increasing, contact is gradually established between the separator and friction discs. As a result, the transmitted torque increases, while the relative velocity $n_{\text {rel }}(t)$ decreases. The clutch is fully engaged when the relative velocity reaches zero value for the first time at the lockup time instant. For an ideal relative velocity signal, the lockup time instant $t_{l}$ can be formulated in a similar way as in Equation (1):

$$
t_{l}=\min \left\{\forall t \in \mathbb{R}: \quad n_{r e l}(t)=0\right\} .
$$

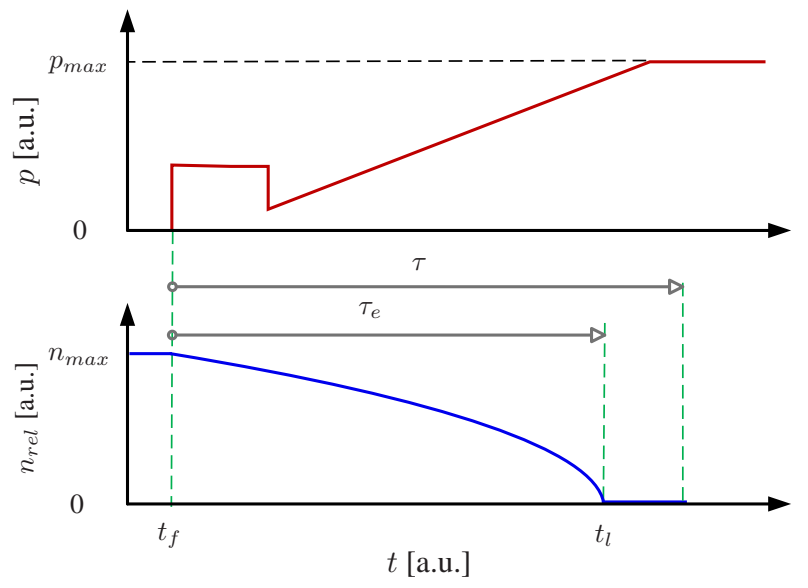

Figure 2. A graphical illustration of how to capture the relative velocity signal of interest. The upper and lower figures respectively denote the typical pressure and the raw relative velocity signal. Note that a.u. is the abbreviation of arbitrary unit.

In practice, data obtained from measurements are normally discretized with some possible noise. As a result, the estimation of $t_{f}$ and $t_{l}$ is no longer straightforward. To deal with this, two simple algorithms based on the linear interpolation technique are developed. The interpolation algorithms identify both time instants $t_{f}$ and $t_{l}$, when limit values for the pressure $p^{l i m}$ and the velocity $n_{r e l}^{l i m}$ are exceeded, as illustrated in Figure 3. The limit values are chosen such that they are higher than the floor noise level of pressure and velocity, i.e. $p^{\text {lim }}>\max \left\{n_{p}^{f}\right\}$ and $n_{r e l}^{\text {lim }}>\max \left\{n_{n}^{f}\right\}$, with $n_{p}^{f}$ and $n_{n}^{f}$ respectively denoting the pressure and the velocity floor noise. The algorithms are discussed in the subsequent paragraphs.

Assume that each measured signal is discretized with sampling time $T_{s}$ and number of sampling points $N$. By knowing that a discrete signal is a set of finite sampling points, it is therefore convenient to treat a measured signal as a vector. Let two vectors $\boldsymbol{P}$ and $\boldsymbol{V}$ be respectively denoting the discrete raw pressure and velocity signal vectors with $N$ sample points. Hence, these $N$-dimensional vectors can be expressed

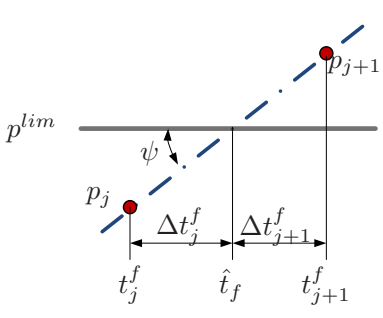

(a)

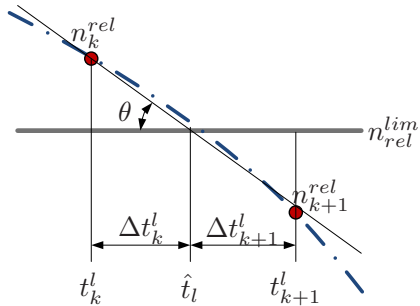

(b)
Figure 3. A graphical representation illustrating the estimation of (a) the reference time instant $\hat{t}_{f}$ based on the pressure signal $p$ and (b) the lockup time instant $\hat{t}_{l}$ based on the relative velocity signal $n_{\text {rel }}$.

in a matrix format as follows:

$$
\begin{array}{r}
\boldsymbol{P}=\left[p_{1}, p_{2}, \ldots p_{j}, \ldots, p_{N-1}, p_{N}\right]^{T}, \\
\boldsymbol{V}=\left[n_{1}^{r e l}, n_{2}^{r e l}, \ldots, n_{k}^{r e l}, \ldots, n_{N-1}^{r e l}, n_{N}^{r e l}\right]^{T} .
\end{array}
$$

Equations (1) and (2) do not apply anymore for discrete and noisy (actual) signals. Nevertheless, these two equations can be adapted in order to estimate the reference time instant $\hat{t}_{f}$ and lockup time instant $\hat{t}_{l}$ from actual signals. The index $j$ of the instantaneous applied pressure $p_{j}$ just before crossing the $p^{l i m}$, see Figure 3(a), is computed with the following equation:

$$
j=\min \left\{\forall j \in \mathbb{Z}: \quad\left(p_{j}-p^{l i m}\right) *\left(p_{j+1}-p^{l i m}\right)<0\right\} .
$$

Based on the index $j$, the estimated reference time instant $\hat{t}_{f}$ can be computed as follows, see Figure 3(a):

$$
\hat{t}_{f}=t_{j}^{f}+\Delta t_{j}^{f} \quad \text { or } \quad \hat{t}_{f}=t_{j+1}^{f}-\Delta t_{j+1}^{f}
$$

with:

$$
\begin{array}{r}
t_{j}^{f}=(j-1) T_{s} \quad \text { and } \quad t_{j+1}^{f}=j T_{s}, \\
\Delta t_{j}^{f}=\left(\frac{p^{l i m}-p_{j}}{p_{j+1}-p_{j}}\right) T_{s}, \\
\Delta t_{j}^{f}=\left(\frac{p_{j+1}-p^{l i m}}{p_{j+1}-p_{j}}\right) T_{s} .
\end{array}
$$

The index $k$ of the relative velocity signal $n_{r e l}^{k}$ just before crossing the $n_{r e l}^{\lim }$ is computed with the following equation:

$k=\min \left\{\forall k \in \mathbb{Z}: \quad\left(n_{k}^{r e l}-n_{r e l}^{l i m}\right) *\left(n_{k+1}^{r e l}-n_{r e l}^{l i m}\right)<0\right\}$.

In similar way, based on the index $k$, the estimated lockup time instant $\hat{t}_{l}$ can be computed as follows:

$$
\hat{t}_{l}=t_{k}^{l}+\Delta t_{k}^{l} \quad \text { or } \quad \hat{t}_{l}=t_{k+1}^{l}-\Delta t_{k+1}^{l}
$$


with:

$$
\begin{array}{r}
t_{k}^{l}=(k-1) T_{s} \quad \text { and } t_{k+1}^{l}=k T_{s}, \\
\Delta t_{k}^{l}=\left(\frac{n_{r e l}^{k}-n_{r e l}^{l i m}}{n_{r e l}^{k}-n_{r e l}^{k+1}}\right) T_{s}, \\
\Delta t_{k}^{l}=\left(\frac{n_{r e l}^{l i m}-n_{r e l}^{k+1}}{n_{r e l}^{k}-n_{r e l}^{k+1}}\right) T_{s} .
\end{array}
$$

\subsection{Feature extraction}

Formal definitions of the developed features (engagement duration, Euclidean distance and Spectral Angle Mapper distance) and the mathematical expressions to compute them are discussed in this subsection. The first two features are dimensional quantities while the third one is dimensionless. The first two features are normalized such that they become dimensionless quantities and are in the same order of magnitude as of the third feature.

\subsubsection{Engagement Duration}

By definition, the engagement duration $\tau_{e}$ is referred to as the time interval between the lockup time instant $t_{l}$ and the reference time instant $t_{f}$, as graphically illustrated in Figure 2. However, these two ideal time instants cannot be obtained from the actual measurement data. Instead, these time instants are estimated based on the procedure previously described. Once both estimates time instants $\hat{t}_{f}$ and $\hat{t}_{l}$ have been determined, the estimate engagement duration $\hat{\tau}_{e}$ can then be simply computed as follows:

$$
\hat{\tau}_{e}=\hat{t}_{l}-\hat{t}_{f} .
$$

Without loss of generality, $\hat{\tau}_{e}$ can be normalized with respect to the engagement duration measured at the first cycle (initial condition) $\hat{\tau}_{e}^{r}$, according to the following equation:

$$
\bar{\tau}_{e}=\frac{\hat{\tau}_{e}-\hat{\tau}_{e}^{r}}{\hat{\tau}_{e}^{r}},
$$

where $\bar{\tau}_{e}$ denotes the dimensionless engagement duration.

\subsubsection{Dissimilarity Measures}

A dissimilarity measure is a metric that quantifies the dissimilarity between objects. For the sake of condition monitoring, the dissimilarity measure between an object that represents an arbitrary condition and the reference object that represents a healthy condition, can be treated as a feature. Thus, the dissimilarity measure between two identical objects is (close to) zero; the dissimilarity measure between two non-identical objects on the other hand is not zero. Here, the object refers to the relative velocity signal. Two dissimilarity measures, namely the Euclidean distance and the Spectral Angle Mapper (SAM) distance, are considered in this paper because of their computational simplicity.
The basic principle behind the dissimilarity approach is that the measured signals of interest are treated as vectors. Let $\boldsymbol{X}$ be a $K$ dimensional vector, $x_{i}, i=1,2, \ldots, K$, denoting the discrete signal of the relative velocity measured in an initial (healthy) condition and $\boldsymbol{Y}$ be a $K$ dimensional vector, $y_{i}, i=1,2, \ldots, K$, denoting the discrete signal of the relative velocity measured in an arbitrary condition. The vector $\boldsymbol{X}$ representing a healthy condition is referred to as the "baseline". Note that one can also take the average of several relative velocities measured in the healthy condition as the baseline, in order to increase the confidence level and accuracy.

The Euclidean distance $\left(D_{E}\right)$ between the vectors $\boldsymbol{X}$ and $\boldsymbol{Y}$ is defined as:

$$
D_{E}(\boldsymbol{X}, \boldsymbol{Y})=\sqrt{\sum_{i=1}^{K}\left(x_{i}-y_{i}\right)^{2}} .
$$

For convenience, the Euclidean distance $D_{E}$ can be normalized in accordance with the following equation:

$$
\bar{D}_{E}(\boldsymbol{X}, \boldsymbol{Y})=\frac{D_{E}(\boldsymbol{X}, \boldsymbol{Y})}{x_{1} \sqrt{K}},
$$

where $\bar{D}_{E}$ denotes the dimensionless Euclidean distance and $x_{1}=\max \{\boldsymbol{X}\}>0$ denotes the maximum value of the baseline, i.e. the initial relative velocity in healthy condition. This way, the dimensionless Euclidean distance $\bar{D}_{E}$ is bounded between 0 and 1, see Appendix A.

The SAM distance is a measure of the angle between two vectors and is therefore dimensionless. Mathematically, the SAM distance $\bar{D}_{S A M}$ between the vectors $\boldsymbol{X}$ and $\boldsymbol{Y}$ is defined as:

$$
\bar{D}_{S A M}(\boldsymbol{X}, \boldsymbol{Y})=\cos ^{-1}\left(\frac{\sum_{i=1}^{K} x_{i} y_{i}}{\sqrt{\sum_{i=1}^{K} x_{i}^{2}} \sqrt{\sum_{i=1}^{K} y_{i}^{2}}}\right) .
$$

Recall that the distance from an object to itself is zero and that a distance is always non-negative. To compute the two dissimilarity measures, a baseline $\boldsymbol{X}$, i.e. the signal of interest in healthy condition, is required and the signal of interest in an arbitrary condition $\boldsymbol{Y}$ must have the same size with the baseline. In this paper, the signal of interest from the first duty cycle is taken as the baseline since it can represent the healthy condition.

\section{EXPERIMENTS}

Service life data of wet friction clutches are required for the evaluation of the developed condition monitoring system. In order to obtain the clutch service life data in a reasonable period of time, the concept of an accelerated life test (ALT) is applied in this study. For this purpose, a fully instrumented SAE\#2 test setup designed and built by the industrial partner, Dana Spicer Off Highway Belgium, was made available. 
According to the standard of the Society of Automotive Engineer (SAE) (i.e. SAE J2489) (SAE-International, 2012), an SAE\#2 test setup is used to evaluate the friction characteristics of automatic transmission clutches with automotive transmission fluids (ATFs). It can also be used to conduct durability tests on wet friction clutch systems and to evaluate the performance variation as a function of the number of duty cycles. Normally, a typical SAE\#2 test setup is equipped with a flywheel driven by an electric motor and the kinetic energy of this wheel is dissipated in a tested clutch (Ost et al., 2001).

An ALT can be realized by means of applying a higher mechanical energy to a tested clutch compared to the amount of energy transmitted by a clutch in normal operation. The energy level is normally adjusted by changing the initial relative velocity and/or the inertia of input and output flywheels. In this study, the ALTs were conducted on some wet friction clutches with different friction materials using a fully instrumented SAE\#2 test setup. During the tests, all the clutches were lubricated with the same Automatic Transmission Fluid (ATF). The used SAE\#2 test setup and the proposed ALT procedure are discussed in the following subsections.

\subsection{SAE\#2 test setup}

The SAE\#2 test setup used in the experiments, as depicted in Figure 4, consists of three main systems, namely: driveline, control and measurement system. The driveline comprises several components: an AC motor for driving the input shaft (1), an input velocity sensor (2), an input flywheel (3), a clutch pack (4), a torque sensor (5), output flywheel (6), an output velocity sensor (7), an AC motor for driving the output shaft (8), a hydraulic system (11-20) and a heat exchanger (21) for cooling the outlet ATF. An integrated control and measurement system (22) is used for controlling the ATF pressure (both for lubrication and actuation) to the clutch and for the initial velocity of both input and output flywheels as well as for measuring all relevant dynamic signals. It should be mentioned here that both velocity sensors are Hall-effect encoders sensing gears with the teeth number of 51. This means that, the resolution of the used rotational velocity sensors is 51 pulses per revolution.

\subsection{Test specification}

To experimentally verify the developed condition monitoring method for wet friction clutches in various conditions and configurations, a test scenario was designed. The general specification of the test scenario is given in Table 1. Five experiments were conducted in this study wherein a different clutch pack was used for each experiment. The energy applied to each clutch pack in the first four tests is set to a relatively high level; while the energy applied in the last test is set at a lower level, see Table 2. In terms of design, all the used clutch packs are identical, only the friction material

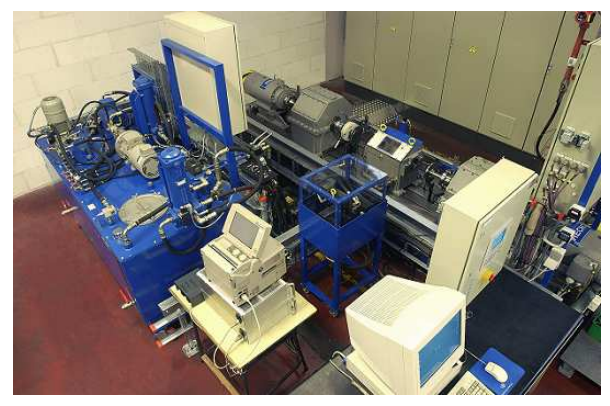

(a)

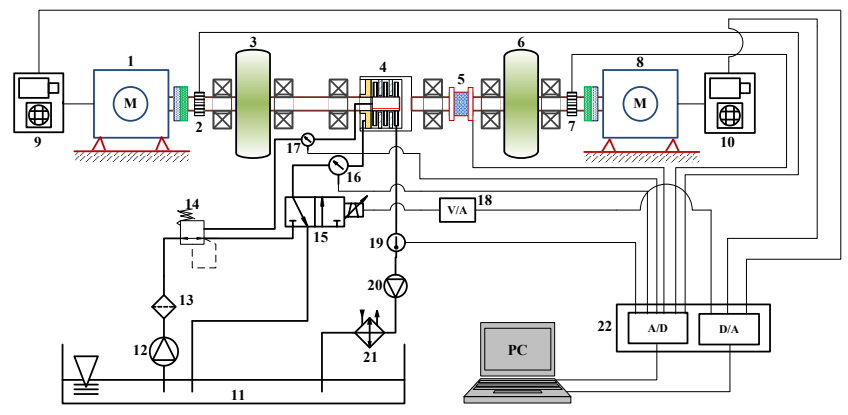

(b)

Figure 4. The SAE\#2 test setup used in the study, (a) photograph and (b) scheme, courtesy of Dana Spicer Off Highway Belgium.

is different for each clutch pack, see Table 2. Lining materials of the friction discs used in all the tests are paper-based type while the materials of all the separator discs are steel. It should be noted that all the used friction discs, separator discs and ATF are commercial ones which can be found in the market. In all the tests, the inlet temperature and flow of the ATF were kept constant, see Table 1. Additionally, one can see in the table that the inertia of the input flywheel (drum-side) is lower than that of the output flywheel (hub-side).

\begin{tabular}{lc}
\hline Number of clutch packs to be tested & 5 \\
Number of friction discs in the clutch assembly & 8 \\
Inner diameter of friction disc $\left(d_{i}\right)[\mathrm{mm}]$ & 115 \\
Outer diameter of friction disc $\left(d_{o}\right)[\mathrm{mm}]$ & 160 \\
ATF & John Deere J20C \\
Lubrication flow [liter/minute] & 18 \\
Inlet temperature of ATF $\left[{ }^{\circ} \mathrm{C}\right]$ & 85 \\
Output flywheel inertia $\left[\mathrm{kgm}^{2}\right]$ & 3.99 \\
Input flywheel inertia $\left[\mathrm{kgm}^{2}\right]$ & 3.38 \\
Sampling frequency $[\mathrm{kHz}]$ & 1 \\
\hline
\end{tabular}

Table 1. General test specification.

\subsection{Test procedure}

Before an ALT is carried out to a wet friction clutch, a run-in test (lower energy level) is first conducted for 100 duty cycles 


\begin{tabular}{cllc}
\hline $\begin{array}{c}\text { Clutch } \\
\text { pack }\end{array}$ & Friction disc & Separator disc & $\begin{array}{c}\text { Initial relative } \\
\text { rotational velocity [rpm] }\end{array}$ \\
\hline 1 & Dynax & Miba Tyzack & 3,950 \\
2 & Raybestos I & Miba Tyzack & 3950 \\
3 & Raybestos II & Miba Tyzack & 3950 \\
4 & Wellman & Miba Tyzack & 3950 \\
5 & Raybestos III & Miba Tyzack & 2950 \\
\hline
\end{tabular}

Table 2. ALT specifications.

in order to stabilize the contact surface. The run-in test procedure is in principle the same as the ALT procedure, but the initial relative rotational velocity of the run-in tests is lower than that of the ALTs. Figure 5 illustrates a duty cycle of the ALT that is carried out as follows. Initially, while both input flywheel (drum-side) and output flywheel (hub-side) are rotating at respective speeds in opposite direction, the two motors are powered-off and the pressurized ATF is simultaneously applied to a clutch pack at time instant $t_{f}$. The oil thus actuates the clutch piston, pushing the friction and separator discs towards each other. This occurs during the filling phase between the time instants $t_{f}$ and $t_{a}$. While the applied pressure is increasing, contact is gradually established between the separator and friction discs which results in an increase of the transmitted torque and a simultaneous decrease of the relative velocity. Finally, the clutch is completely engaged when the relative velocity reaches zero at the lockup time instant $t_{l}$. As the inertia and the respective initial speed of the output flywheel (hub-side) are higher than those of the input flywheel, after $t_{l}$, both flywheels rotate together in the same direction as the output flywheel, see Figure 5. In order to prepare for the forthcoming duty cycle, both driving motors are braked at the time instant $t_{b}$, such that the driveline can stand still for a while.

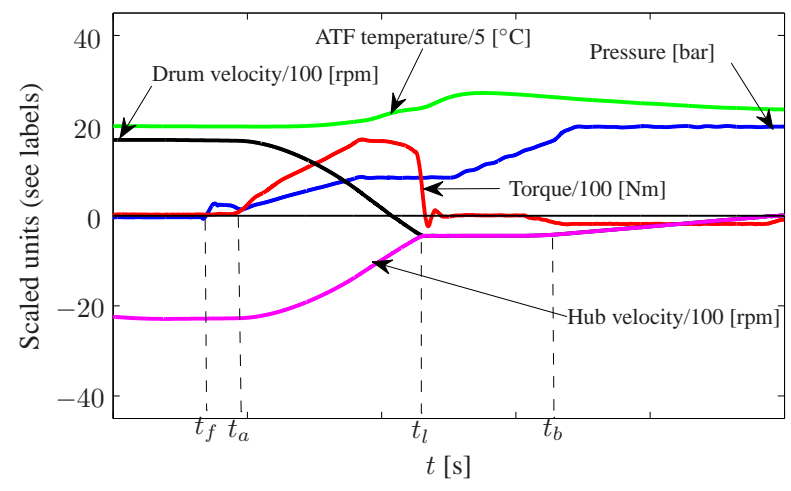

Figure 5. A representative duty cycle of wet friction clutches. Note that the transmitted torque drops to zero after the lockup time instant $t_{l}$ because there is no external load applied during the test.

It is known that the normal load (pressure) has a significant effect on the frictional characteristics. This means the pressure applied to wet friction clutches has also a significant effect on the engagement behavior which in turn influences the signal profile of the relative velocity. Since only the effect of the clutch degradation on the change of the relative velocity signal is of interest, the pressure signal applied to the clutch in each test is therefore kept the same.

The ALT procedure discussed above is continuously repeated until a given total number of duty cycles is attained. For the sake of time efficiency in measurement, all the ALTs are performed for 10000 duty cycles. Moreover, the ATF is continuously filtered, such that it is reasonable to assume that the used ATF has not degraded during all the tests.
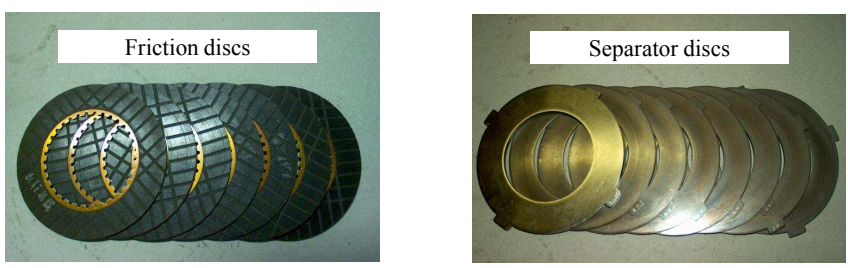

Figure 6. Friction and separator discs after 10000 duty cycles, courtesy of Dana Spicer Off Highway Belgium.

\section{RESUlts AND Discussion}

Figure 6 shows the photographs of friction and separator discs of a wet friction clutch after 10000 duty cycles, taken from the first clutch pack. From the figure it can be seen that the surfaces of the friction discs have become smooth and glossy. Nevertheless, it is evident that the separator discs are still in good condition.

Figure 7 shows the comparisons of the optical images and the surface profile of the friction material before and after the ALT. The images are captured using a Zeiss microscope and the surface profiles are measured along the sliding direction using a Taylor Hobson Talysurf profilometer. It can be seen in the figure that the surface of the friction material has become smooth and glossy and the clutch is therefore considered to have failed. The change of the color and the surface topography of the friction material is known as a result of the glazing phenomenon that is believed to be caused by a combination of adhesive wear and thermal degradation (Gao $\&$ Barber, 2002). Due to these two mechanisms, the surface pores of the friction material are blocked by the deposition of debris particles and/or the deposition of the ATF products.

Without loss of generality, energy density that is defined as the transmitted energy per unit of total contact area is introduced here for comparing the degradation rate of the tested clutches with different test conditions. The transmitted en- 

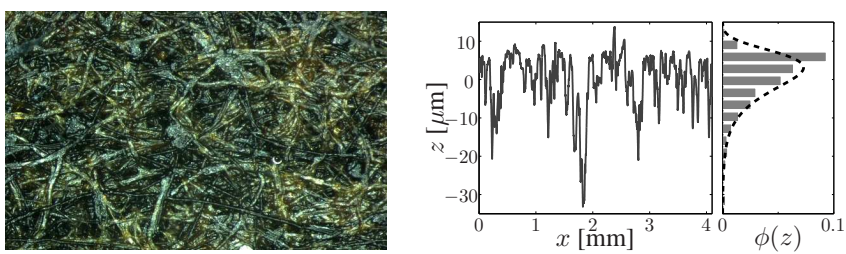

(a)
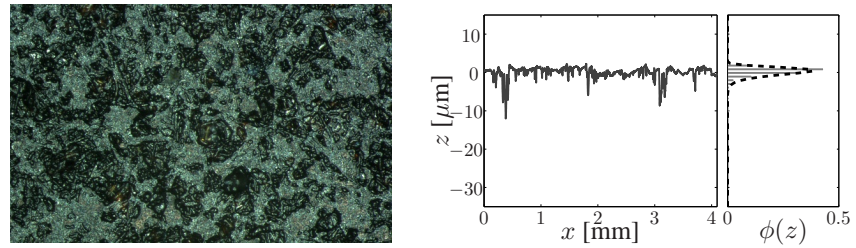

(b)

Figure 7. Comparison of the friction material before and after the ALT of 10000 duty cycles. (a) optical image (left) and the corresponding surface profile (right) of the friction material before the test, (b) optical image (left) and the corresponding surface profile (right) of the friction material after the test. Notice that $z$ denotes the displacement of the profilometer stylus in Z-axis (perpendicular to the surface), $x$ denotes the displacement of the profilometer stylus in $\mathrm{X}$-axis (along the sliding direction) and $\phi(z)$ denotes the probability distribution function of the surface profile.

ergy $E_{c y c l e}$ at a given duty cycle is computed as follows:

$$
E_{\text {cycle }}=\int_{t_{e}}^{t_{l}} M \omega_{r e l} d t,
$$

where $M$ denotes the transmitted torque and $\omega_{r e l}=\frac{2 \pi n_{r e l}}{60}$ denotes the relative velocity in $\mathrm{rad} / \mathrm{s}$. Hence, the energy density $\mathscr{E}_{c y c l e}$ transmitted by a wet friction clutch at a given duty cycle can be computed as follows:

$$
\mathscr{E}_{\text {cycle }}=\frac{E_{\text {cycle }}}{N_{f} \mathscr{A}_{f}}
$$

where $N_{f}$ is the number of friction faces and $\mathscr{A}_{f}$ is the apparent contact area between friction disc and separator disc. By applying Equations (14) - (15) to the measured torque and relative velocity, the energy density of each test can be computed as presented in Table 3. As expected, it can be clearly seen from the table that the energy density $\mathscr{E}_{c y c l e}$ applied to the fifth clutch pack is lower than (approximately half of) that of other clutch packs.

The remainder of this section is structured as follows. First, the COFs of all the tested clutches are computed and their characteristics during the clutch service life are evaluated and discussed. Afterwards, the features proposed in this paper, i.e. the dimensionless engagement time and dimensionless dissimilarity measures, are extracted from the relative velocity signals. Finally, the proposed features are compared and evaluated with the mean COF which has been considered, as mentioned in the introductory section, as a reference feature.

\begin{tabular}{cc}
\hline Test & $\mathscr{E}_{\text {cycle }} \times 10^{-6}\left[\mathrm{Joule} / \mathrm{m}^{2}\right]$ \\
\hline 1 & 1.068 \\
2 & 1.068 \\
3 & 1.068 \\
4 & 1.068 \\
5 & 0.584 \\
\hline
\end{tabular}

Table 3. The energy density $\mathscr{E}_{c y c l e}$ applied in the ALTs.

\subsection{COF characteristic during the service life of the tested clutches}

As previously stated in Section 1, the COF has been used for many years to monitor and evaluate the condition of wet friction clutches. Due to its strong correlation to the nature of clutch degradation, it is therefore reasonable to employ the $\mathrm{COF}$ as a reference feature for evaluating and justifying the relevance of the proposed features with respect to the progression of the clutch degradation. At a given duty cycle, the instantaneous $\mathrm{COF} \mu$ of a wet friction clutch can be computed according to the following equation (Ost et al., 2001):

$$
\mu=\frac{3 M\left(r_{o}^{2}-r_{i}^{2}\right)}{2 N_{f} F_{a}\left(r_{o}^{3}-r_{i}^{3}\right)},
$$

where $r_{o}$ is the outer radius of friction disc, $r_{i}$ is the inner radius of friction disc, $N_{f}$ is the number of friction faces and $F_{a}$ is the axial force applied to the clutch. The applied force can be estimated based on the applied pressure $p$ and the force of the returning spring $F_{s}$, i.e. $F_{a} \approx p A_{p}-F_{s}$, with $A_{p}$ denoting the area of the piston.

Suppose that the spring force and friction disc geometry are given, the instantaneous COFs of all the tested clutch packs can be computed by applying Equation (16) to the experimental data (the measured torque and pressure signals). The spring force is determined here based on the deformation of the returning spring, when the piston and all the discs make contact, with respect to its rest position.

In order to quantify the global characteristic of the COF of a wet friction clutch, the mean COF $\mu_{m}$ as proposed in (Ost et al., 2001) can be applied for this purpose. For one duty cycle, this quantity is defined as follows:

$$
\mu_{m}=\frac{1}{\left(t_{l}-t_{e}\right)} \int_{t_{e}}^{t_{l}} \mu d \tau .
$$

For a discrete data set, i.e. $\mu=\left[\mu_{1}, \mu_{2}, \ldots, \mu_{j}, \ldots, \mu_{L}\right]$, the mean COF computed with Equation (17) can be rewritten as follows:

$$
\mu_{m}=\frac{1}{L} \sum_{j=1}^{j=L} \mu_{j},
$$

with $L$ denoting the dataset length of the instantaneous (discrete) COF $\mu_{j}$ and indexes $j=1$ and $j=L$ correspond to the time instant $t_{e}$ and $t_{l}$, respectively. For convenience, the 
$\mu_{m}$ can be normalized according to the following equation:

$$
\delta \hat{\mu}_{m}=\frac{\mu_{m}-\mu_{m}^{r}}{\mu_{m}^{r}},
$$

with $\delta \hat{\mu}_{m}$ denoting the normalized mean COF and $\mu_{m}^{r}$ denoting the mean COF measured from the first duty cycle.

In Figure 8, the evolution of the mean COFs and the normalized ones are depicted. The dropping mean COFs can be explained as follows. As the degradation progresses, the surface of the friction material becomes smoother and debris particles are possibly entrapped on the surface pores of the friction material. Moreover, the deposition of ATF products may also blockade the surface pores of the friction material. This complex phenomenon is well known as a glazing process ( $\mathrm{Li}$ et al., 2003). As a result of the glazing phenomenon, the ability of the ATF to escape from the approaching contact surfaces decreases. In this particular situation, the ATF stays between the contacting surfaces which hampers the occurrence of surface to surface contact corresponding to the boundary lubrication regime. Thus, the occurring friction is mainly controlled by the ATF resulting in lower COFs. In addition to this, the mechanical properties of the friction material change, e.g. reduction of the shear strength (Maeda \& Murakami, 2003), which may have an additional effect on the COFs reduction. By considering that the ATF has not signif-
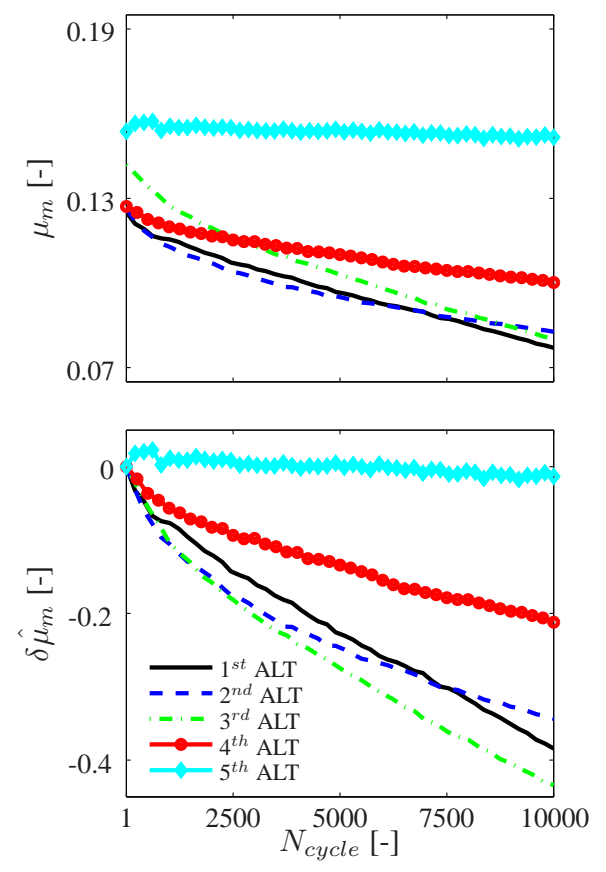

Figure 8. Evolution of the mean $\mathrm{COF}$ and its normalized value during the lifetime of the tested clutches.

icantly degraded during the tests (which is the case in this study), the progressive change of the COFs implies that all the tested friction materials have degraded to a certain extent. The level of friction material degradation is not only dependent on the amount of input energy, but also the design, e.g. material durability. As can be seen in Figure 8, the effect of the used friction material on the COF evolution can be observed. Despite the same energy level, the mean COF reduction of the fourth clutch pack with the Wellman friction material is less than that of the first three clutch packs. It can be seen in the figure that the dimensionless mean COF reduction after 10000 cycles of this particular friction material is approximately half of the others conducted at the same energy level (Dynax, Raybestos I and Raybestos II, see Table 2). Accordingly, one may conclude that the Wellman product used in the $4^{\text {th }}$ ALT is more durable than the other friction materials tested in the study. In addition, the effect of energy level can also be observed from the data (compare the $5^{\text {th }}$ ALT with the other tests). As expected, the lower the energy level the smaller the COF alteration will be.

\subsection{Experimental verification of the developed condition monitoring method}

The pressure signals obtained from the measurements are plotted in the left panels of Figures 9 - 13. The figures show that the pressure signals applied to a tested clutch during the ALT are relatively identical. This suggests that the effect of the pressure variation on the change of the relative velocity signal during the clutch lifetime can be assumed negligible.

Different from the fifth ALT, the service life relative velocity signals obtained from the first four ALTs show noticeable changes; see Figures 9 - 12. These noticeable changes can be explained as follows. In the first four tests, the initial velocity is set at high value which gives a large amount of energy to the clutches to be tested. A higher energy level applied on a clutch implies that a higher degradation rate occurs in the friction material. As a result, the frictional characteristics change significantly which is reflected by the noticeable change of the relative velocity signal. Accordingly, it can be deduced that with the same operational condition and identical clutches, the shape of the relative velocity signal profile of clutches is strongly dependent on its initial value, see Figures 11 and 13. As was expected, the higher the initial relative velocity is, the longer the engagement duration will be and vice versa.

In order to quantify the change of the relative velocity signals during the clutches service life, the proposed features as have been introduced in Section 2 are extracted from the service life data using Equations (10), (12) and (13). The plots of these features in function of the clutches service life are depicted in Figure 14. The figure clearly show the progress of clutch degradation which is embodied in the features evolution. This observation implies that the proposed features are relevant to monitor the progression of clutch degradation. 

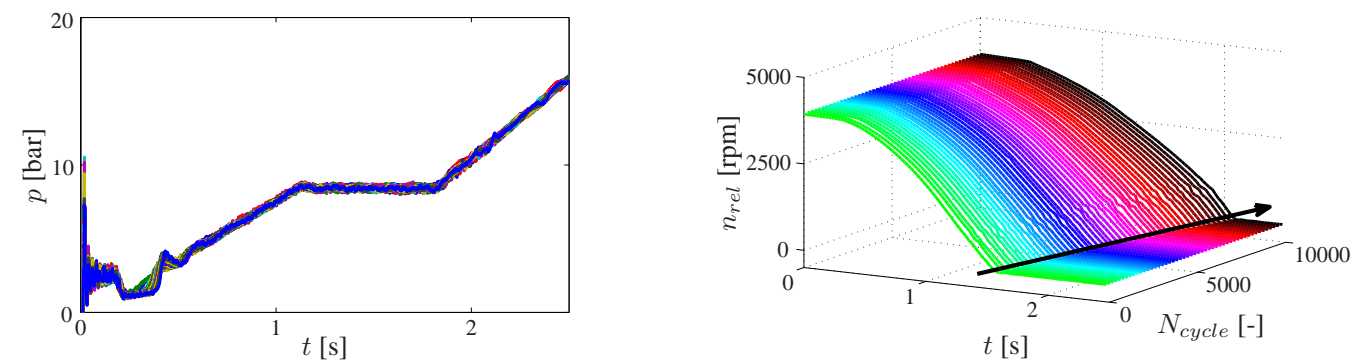

Figure 9. Pressure signals (left) and relative velocity signals (right) obtained from the first ALT.
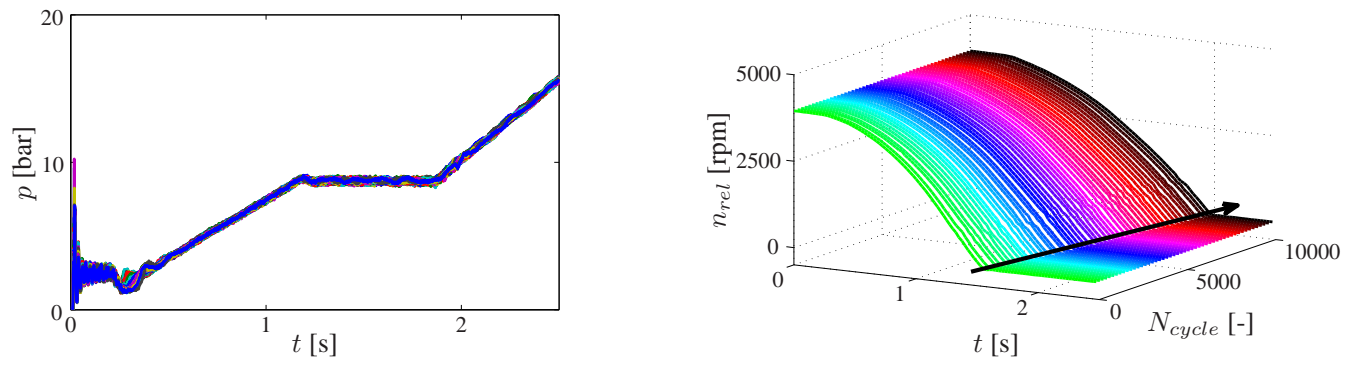

Figure 10. Pressure signals (left) and relative velocity signals (right) obtained from the second ALT.
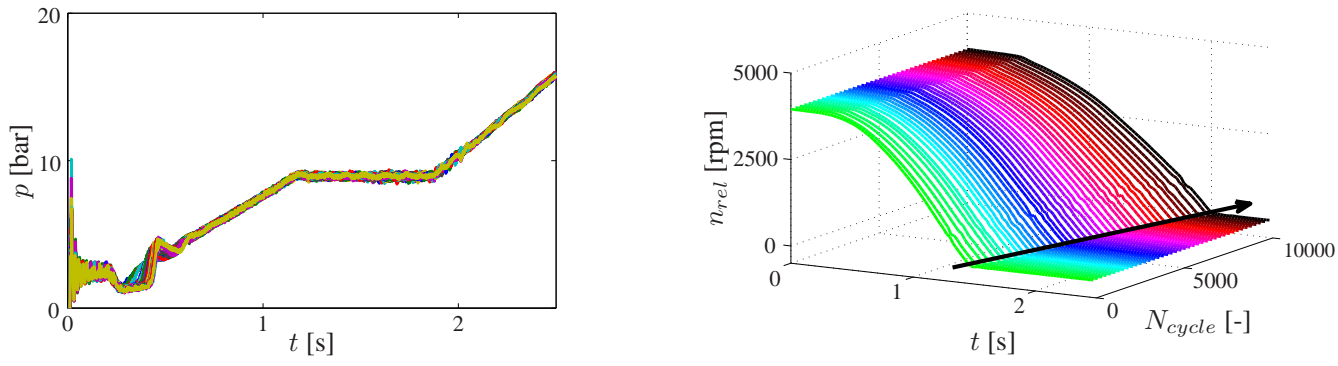

Figure 11. Pressure signals (left) and relative velocity signals (right) obtained from the third ALT.
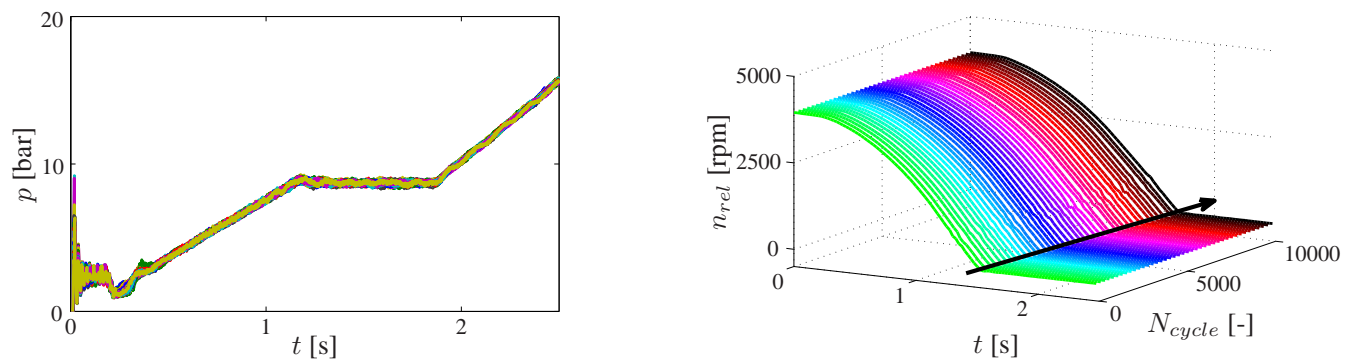

Figure 12. Pressure signals (left) and relative velocity signals (right) obtained from the fourth ALT.
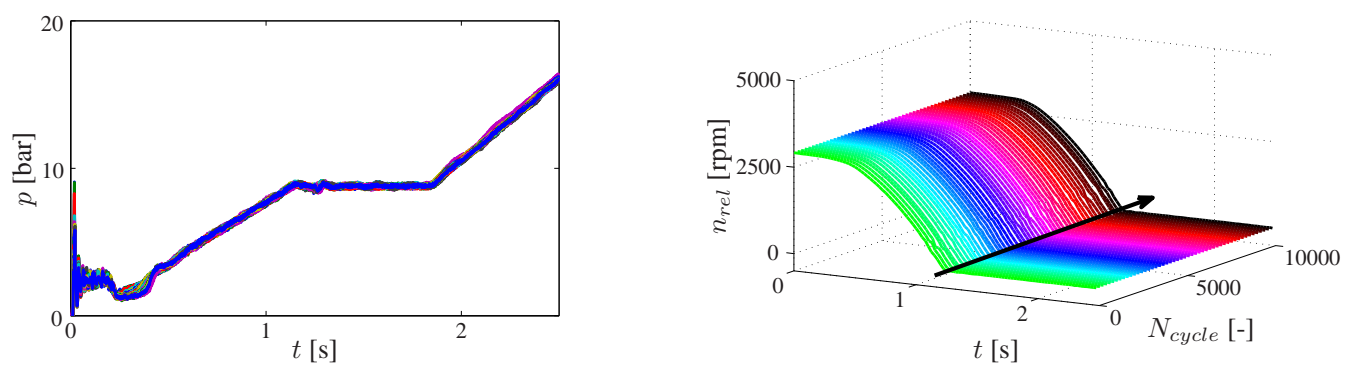

Figure 13. Pressure signals (left) and relative velocity signals (right) obtained from the fifth ALT. 
The increasing dimensionless engagement duration as depicted in the upper panel of Figure 14 implies that the COF drops as the degradation progresses, see Figure 8, which confirms the experimental data available in the literature (Matsuo \& Saeki, 1997; Ost et al., 2001; Maeda \& Murakami, 2003; Fei et al., 2008). For the same operating conditions (e.g. the same applied pressure to the piston clutch), as has been mentioned previously, the dropping COF consequently lowers the transmitted torque during the clutch service life. When the transmitted torque becomes lower, the relative velocity decreases more slowly from its initial value to zero value.
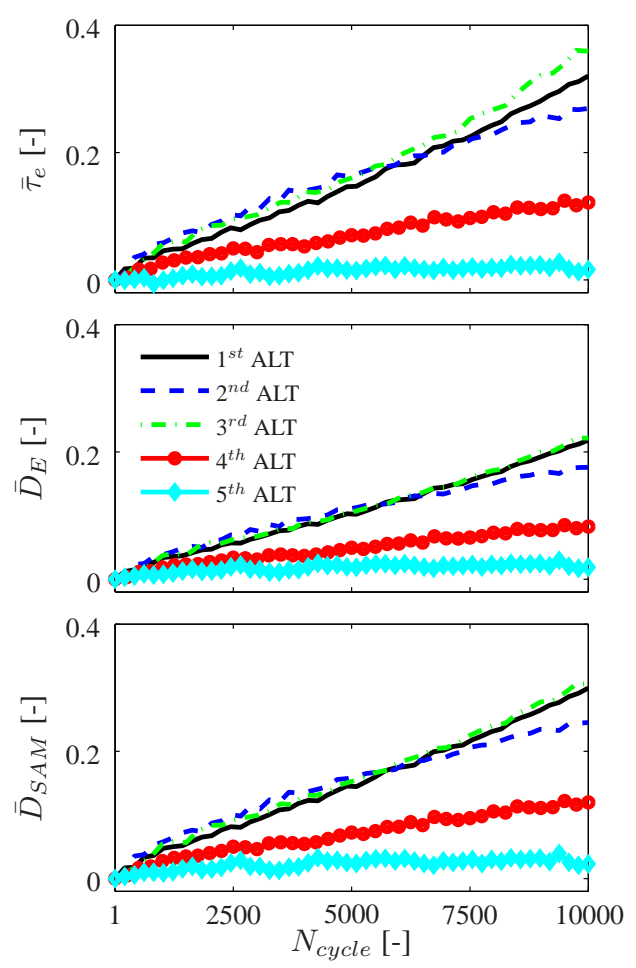

Figure 14. Evolution of the proposed dimensionless features during the lifetime of the tested clutches.

Since the relative velocity signal of interest obtained from the first duty cycle is taken as the baseline as discussed previously in Section 2, the dissimilarity measures at the first cycle are zero as can be seen in the middle and lower panels of Figure 14. When the clutch degradation progresses further, the shape of the relative velocity signal of interest deviates from the baseline which consequently results in an increase of the dissimilarity measures.

Remarkably, all the plots of the proposed features in function of the clutch duty cycles show linearly increasing trends with relatively small variations. The possible explanation for this observations is that all the tests were repeated with the same operational conditions. In practice, the operating conditions can vary and the energy level per duty cycle is typically lower than that applied in this study. Hence, the trends of the proposed features are not necessarily linear and the variations are not necessarily small. Because of possibly varying operating conditions, the trends of the proposed features may exhibit non-linear behaviors with large variations in real situations. In addition to this, the rate of change of the feature values in real-situations is possibly lower than that observed on the ALT results of this study.

\section{Conclusion AND Future Work}

A condition monitoring method for wet friction clutches that can be used in real-life applications has been developed and discussed in this paper. The method is based on monitoring the change of the relative velocity signal measured between the input and output shaft of a clutch. Three dimensionless parameters which are easy to compute, namely the normalized engagement duration, the normalized Euclidean distance and the Spectral Angle Mapper (SAM) distance are proposed in this paper as features for monitoring the condition of wet friction clutches. The developed method suggests that the sensors typically available in automatic transmissions, namely pressure and velocity sensors, can be employed for extracting the proposed features.

Service life data obtained from accelerated life tests (ALTs), carried out using an SAE\#2 test setup on five different paperbased wet friction clutches, are employed in order to evaluate and verify the potential and the relevance of the proposed features for clutch condition monitoring. All the plots of the proposed features in function of the service life of the tested clutches exhibit clear trends, which can be associated with the progress of the clutch degradation. The trends are well correlated with the mean coefficient of friction (COF) which can be considered here as a reference feature. Remarkably, all the trends exhibited by the features proposed in the paper are (monotonically) increasing with relatively small variations. For akin clutches, it has been observed that the evolution of the features extracted from the clutches' life data obtained from the ALTs with a higher energy level, shows a steeper trend (larger slope) compared to the one with lower energy level; where this observation is also consistent with the result of the mean COF. The slope difference leads to a conclusion that clutches tested with higher energy level experience a higher degradation rate than those with lower energy level. Since the proposed features are able to expose the progression of clutch degradation, their relevance for monitoring and assessing the condition of wet friction clutches are justified.

So far, the developed clutch monitoring method has been evaluated and verified on ALTs under controlled environment, where (i) the inlet ATF temperature is controlled at a constant value, (ii) the applied pressure variation is relatively 
small and (iii) the external load is fixed. In practice, the operating condition can vary that may affect the accuracy of the developed monitoring method. The effects of the operational parameter variations on the proposed features need to be further investigated. A profound understanding may allow us to model the feature variations such that an accurate monitoring system can be achieved.

\section{A. ApPEndix}

The Euclidean distance $D_{E}$ between two signals of interest is defined similarly as the Euclidean distance between two vectors $\boldsymbol{X}$ and $\boldsymbol{Y}$ as follows:

$$
D_{E}(\boldsymbol{X}, \boldsymbol{Y})=\sqrt{\sum_{i=1}^{K}\left(x_{i}-y_{i}\right)^{2}}
$$

If Equation (A.1) is imposed in the following form:

$$
\bar{D}_{E}(\boldsymbol{X}, \boldsymbol{Y})=\frac{D_{E}(\boldsymbol{X}, \boldsymbol{Y})}{x_{1} \sqrt{K}},
$$

then we have:

$$
0 \leq \bar{D}_{E}(\boldsymbol{X}, \boldsymbol{Y}) \leq 1
$$

Therefore $\bar{D}_{E}(\boldsymbol{X}, \boldsymbol{Y})$ can be seen as a normalized version of $D_{E}(\boldsymbol{X}, \boldsymbol{Y})$.

Proof. Let Equation (A.2) be expanded as follows:

$$
\begin{aligned}
\bar{D}_{E}(\boldsymbol{X}, \boldsymbol{Y}) & =\frac{D_{E}(\boldsymbol{X}, \boldsymbol{Y})}{x_{1} \sqrt{K}}, \\
& =\sqrt{\frac{1}{K} \sum_{i=1}^{K} \frac{\left(x_{i}-y_{i}\right)^{2}}{x_{1}^{2}}}, \\
& =\sqrt{\frac{1}{K} \sum_{i=1}^{K}\left(\frac{x_{i}}{x_{1}}-\frac{y_{i}}{x_{1}}\right)^{2}} .
\end{aligned}
$$

Assume that the following properties hold:

$$
\left\{\begin{array}{l}
0 \leq x_{i} \leq x_{1}, \quad i=1, \ldots, K, \\
0 \leq y_{j} \leq x_{1}, \quad j=1, \ldots, K .
\end{array}\right.
$$

From which it follows that:

$$
\left\{\begin{array}{l}
0 \leq \frac{x_{i}}{x_{1}}=\varepsilon_{i} \leq 1, \quad i=1, \ldots, K \\
0 \leq \frac{y_{j}}{x_{1}}=\eta_{j} \leq 1, \quad j=1, \ldots, K .
\end{array}\right.
$$

The inequalities above can be solved as follows:

$$
\begin{array}{rlrl}
-1 & \leq \quad \varepsilon_{i}-\eta_{i} & \leq 1 \\
0 & \leq \quad\left(\varepsilon_{i}-\eta_{i}\right)^{2} & \leq 1 \\
0 & \leq \sum_{i=1}^{K}\left(\varepsilon_{i}-\eta_{i}\right)^{2} & \leq K \\
0 & \leq \frac{1}{K} \sum_{i=1}^{K}\left(\varepsilon_{i}-\eta_{i}\right)^{2} & \leq 1 \\
0 & \leq \sqrt{\frac{1}{K} \sum_{i=1}^{K}\left(\varepsilon_{i}-\eta_{i}\right)^{2}} \leq 1,
\end{array}
$$

\begin{tabular}{|c|c|}
\hline$t$ & time \\
\hline$n_{\text {rel }}$ & relative velocity in rpm \\
\hline$\omega_{\text {rel }}$ & relative velocity in $\mathrm{rad} / \mathrm{s}$ \\
\hline$p$ & pressure \\
\hline$M$ & torque \\
\hline$F_{a}$ & axial force \\
\hline$E_{\text {cycle }}$ & energy transmitted for a given cycle \\
\hline $\mathscr{E}_{\text {cycle }}$ & energy density transmitted for a given cycle \\
\hline $\mathscr{A}_{f}$ & apparent contact area \\
\hline$A_{p}$ & contact area of piston \\
\hline$x$ & $\begin{array}{l}\text { displacement of the profilometer stylus in } \mathrm{X} \text {-axis } \\
\text { along sliding direction }\end{array}$ \\
\hline$z$ & $\begin{array}{l}\text { displacement of the profilometer stylus in Z-axis } \\
\text { perpendicular to the surface }\end{array}$ \\
\hline$\phi(z)$ & $\begin{array}{l}\text { probability distribution function } \\
\text { of the surface profile }\end{array}$ \\
\hline$r_{i}$ & inner radius of friction disc \\
\hline$r_{o}$ & outer radius of friction disc \\
\hline$\mu$ & instantaneous coefficient of friction \\
\hline$\mu_{m}$ & averaged coefficient of friction \\
\hline$t_{f}$ & true reference time instant \\
\hline$t_{l}$ & true lockup time instant \\
\hline$\hat{t}_{f}$ & estimated reference time instant \\
\hline$\hat{t}_{l}$ & estimated lockup time instant \\
\hline$j, k$ & indices \\
\hline$n_{n}^{f}$ & floor noise relative velocity signal \\
\hline$n_{p}^{f}$ & floor noise pressure signal \\
\hline$\tau$ & time record length \\
\hline$\tau_{e}$ & engagement duration \\
\hline$X$ & $\begin{array}{l}\text { vector denoting a discrete relative velocity signal } \\
\text { measured in an initial (healthy) condition }\end{array}$ \\
\hline $\boldsymbol{Y}$ & $\begin{array}{l}\text { vector denoting a discrete relative velocity signal } \\
\text { measured in an arbitrary condition }\end{array}$ \\
\hline $\bar{\tau}_{e}$ & normalized engagement duration \\
\hline $\bar{D}_{E}$ & normalized Euclidean distance \\
\hline $\bar{D}_{S A M}$ & normalized SAM distance \\
\hline$N_{\text {cycle }}$ & number of duty (engagement) cycles \\
\hline$N_{f}$ & number of friction faces \\
\hline
\end{tabular}

which proves that:

$$
0 \leq \bar{D}_{E}(\boldsymbol{X}, \boldsymbol{Y}) \leq 1
$$

\section{ACKNOWLEDGMENT}

All the authors wish to thank Dr. Mark Versteyhe of Dana Spicer Off Highway Belgium for the experimental support.

\section{NOMENCLATURE}




\section{REFERENCES}

Fei, J., Li, H.-J., Qi, L.-H., Fu, Y.-W., \& Li, X.-T. (2008). Carbon-Fiber Reinforced Paper-Based Friction Material: Study on Friction Stability as a Function of Operating Variables. Journal of Tribology, 130(4), 041605.

Gao, H., \& Barber, G. C. (2002). Microcontact Model for Paper-Based Wet Friction Materials. Journal of Tribology, 124(2), 414 - 419.

Guan, J., Willermet, P., Carter, R., \& Melotik., D. (1998). Interaction Between ATFs and Friction Material for Modulated Torque Converter Clutches. SAE Technical Paper, 981098, 245252.

Jullien, A., Meurisse, M., \& Berthier, Y. (1996). Determination of tribological history and wear through visualisation in lubricated contacts using a carbon-based composite. Wear, 194(1 - 2), 116 - 125.

Kruse, F., Lefkoff, A., Boardman, J., Heidebrecht, K., Shapiro, A., Barloon, P., et al. (1993). The spectral image processing system (SIPS) - interactive visualization and analysis of imaging spectrometer data. Remote Sensing of Environment, 44(2-3), 145 - 163.

Li, S., Devlin, M., Tersigni, S., Jao, T.-C., Yatsunami, K., \& Cameron., T. (2003). Fundamentals of Anti-Shudder Durability: Part I - Clutch Plate Study. SAE Technical Paper, 2003-01-1983, 5162.

Maeda, M., \& Murakami, Y. (2003). Testing method and effect of ATF performance on degradation of wet friction materials. SAE Technical Paper, 2003-01-1982, 45 50 .

Matsuo, K., \& Saeki, S. (1997). Study on the change of friction characteristics with use in the wet clutch of automatic transmission. SAE Technical Paper, 972928, 93 - 98.

Nyman, P., Maki, R., Olsson, R., \& Ganemi, B. (2006). Influence of surface topography on friction characteristics in wet clutch applications. Wear, 261(1), 46 - 52. (Papers presented at the 11th Nordic Symposium on Tribology, NORDTRIB 2004)

Ompusunggu, A. P., Janssens, T., Al-Bender, F., Sas, P., \& VanBrussel, H. (2011). Engagement behavior of degrading wet friction clutches. In 2011 IEEE/ASME International Conference on Advanced Intelligent Mechatronics (AIM2011).

Ompusunggu, A. P., Papy, J.-M., Vandenplas, S., Sas, P., \& VanBrussel, H. (n.d.). A novel monitoring method of wet friction clutches based on the post-lockup torsional vibration signal. Mechanical Systems and Signal Processing, submitted after revision.

Ompusunggu, A. P., Papy, J.-M., Vandenplas, S., Sas, P., \& VanBrussel, H. (2009). Exponential data fitting for features extraction in condition monitoring of paperbased wet clutches. In C. Gentile, F. Benedettini, R. Brincker, \& N. Moller (Eds.), The Proceedings of the 3rd International Operational Modal Analysis Conference (IOMAC) (Vol. 1, p. 323-330). Starrylink Editrice Brescia.

Ompusunggu, A. P., Sas, P., VanBrussel, H., Al-Bender, F., Papy, J.-M., \& Vandenplas, S. (2010). Pre-filtered Hankel Total Least Squares method for condition monitoring of wet friction clutches. In The Proceedings of the 7th International Conference on Condition Monitoring and Machinery Failure Prevention Technologies (CM-MFPT). Coxmor Publishing Company.

Ompusunggu, A. P., Sas, P., VanBrussel, H., Al-Bender, F., Papy, J.-M., \& Vandenplas, S. (2011). Normal-mode vibration analysis for condition monitoring of wet friction clutches. In Proceedings of the 24th International Congress on Condition Monitoring and Diagnostic Engineering Management (COMADEM).

Ompusunggu, A. P., Sas, P., VanBrussel, H., Al-Bender, F., \& Vandenplas, S. (2010). Statistical feature extraction of pre-lockup torsional vibration signals for condition monitoring of wet friction clutches. In Proceedings of ISMA2010 Including USD2010.

Ost, W., Baets, P. D., \& Degrieck, J. (2001). The tribological behaviour of paper friction plates for wet clutch application investigated on SAE \# II and pin-on-disk test rigs. Wear, 249(5-6), 361 - 371.

Paclik, P., \& Duin, R. P. W. (2003). Dissimilarity-based classification of spectra: computational issues. RealTime Imaging, 9(4), 237 - 244.

SAE-International. (2012). SAE No. 2 Friction Test Machine Durability Test (Vol. SAE J2489).

\section{BIOGRAPHIES}

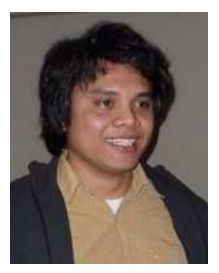

Agusmian Partogi Ompusunggu is a project engineer at Flanders' MECHATRONICS Technology Centre (FMTC), Belgium. His research focuses in condition monitoring, prognostics, vibration analysis and measurement and tribology. He earned his bachelor degree in mechanical engineering (B.Eng) in 2004 from

Institut Teknologi Bandung (ITB), Indonesia and master degree in mechanical engineering (M.Eng) in 2006 from the same technological institute. He is currently pursuing his $\mathrm{PhD}$ degree in mechanical engineering at Katholieke Universiteit Leuven (K.U.Leuven) Belgium. 


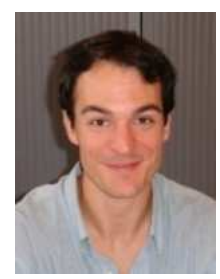

Jean-Michel Papy received a Master Degree in Signal, Image and Acoustics from Paul Sabatier University, Toulouse, France, in 2000 and a PhD degree in Electrical Engineering from the K.U. Leuven, Belgium in 2005. His doctoral work was about the detection of transient signals and exponential data modeling using linear and multi-linear algebra. After his $\mathrm{PhD}$, he has been working as a project engineer at Flanders MECHATRONICS Technology Centre (FMTC), Belgium. His current research interests include modeling of mechanical systems and sensor fusion.

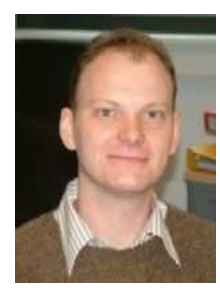

Steve Vandenplas is a program leader at Flanders' MECHATRONICS Technology Centre (FMTC), Belgium. He received his Master's Degree of Electrotechnical Engineer in 1996 from the Vrije Universiteit Brussel (VUB), Belgium. In 2001, he received a $\mathrm{PhD}$ in Applied Science and started to work as $R \& D$ Engineer at Agilent Technologies for one year. Thereafter, he decided to work as a Postdoctoral Fellow at the K.U. Leuven in the Department of Metallurgy and Materials Engineering, in the research group material performance and non-destructive testing (NDT). He has been working at Flanders' MECHATRONICS Technology Centre (FMTC) since 2005, where he is currently leading FMTC's research program on "Monitoring and Diagnostics". His main interests are on machine diagnostics and condition based maintenance (CBM).

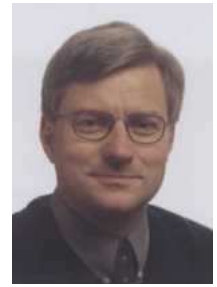

Paul Sas is a full professor at the Department of Mechanical Engineering of Katholieke Universiteit Leuven (K.U.Leuven), Belgium. He received his master and doctoral degree in mechanical engineering from K.U.Leuven. His research interest comprise numerical and experimental techniques in vibroacoustics, active noise and vibration control, noise control of machinery and vehicles, structural dynamics and vehicle dynamics. He is currently leading the noise and vibration research group of the Department of Mechanical Engineering at K.U.Leuven.

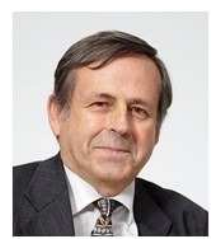

Hendrik Van Brussel is an emeritus professor at the Department of Mechanical Engineering of Katholieke Universiteit Leuven (K.U.Leuven), Belgium. $\mathrm{He}$ obtained the degree of Technical Engineer in mechanical engineering from the Hoger Technisch Instituut in Ostend, Belgium in 1965 and an engineering degree in electrical engineering at M.Sc level from K.U.Leuven. In 1971 he got his $\mathrm{PhD}$ degree in mechanical engineering, also from K.U.Leuven. From 1971 until 1973 he was establishing a Metal Industries Development Center in Bandung, Indonesia and he was an associate professor at Institut Teknologi Bandung (ITB), Indonesia. He was a pioneer in robotics research in Europe and an active promoter of the mechatronics idea as a new paradigm in machine design. He has published more than 200 papers on different aspects of robotics, mechatronics and flexible automation. His research interests shifted towards holonic manufacturing systems and precision engineering, including microrobotics. $\mathrm{He}$ is Fellow of SME and IEEE and in 1994 he received a honorary doctor degree from the 'Politehnica' University in Bucharest, Romania and from RWTH, Aachen, Germany. $\mathrm{He}$ is also a Member of the Royal Academy of Sciences, Literature and Fine Arts of Belgium and Active Member of CIRP (International Institution for Production Engineering Research). 